\title{
BMJ Open Quality Improving antimicrobial stewardship in the outpatient department of a district general hospital in Sierra Leone
}

\author{
David Hamilton, ${ }^{1,2}$ Ian Bugg ${ }^{3}$
}

To cite: Hamilton D, Bugg I. Improving antimicrobial stewardship in the outpatient department of a district general hospital in Sierra Leone. BMJ Open Quality 2018;7:e000495. doi:10.1136/ bmjoq-2018-000495

Received 8 August 2018 Revised 12 November 2018 Accepted 17 November 2018

\section{Check for updates}

(C) Author(s) (or their employer(s)) 2018. Re-use permitted under CC BY-NC. No commercial re-use. See rights and permissions. Published by BMJ.

${ }^{1}$ Critical Care, Northern Care Alliance, Manchester, UK ${ }^{2}$ Masanga Medical Research Unit, Masanga Hospital, Tonkolili District, Sierra Leone

${ }^{3}$ Humanitarian Studies Department, Liverpool School of Tropical Medicine, Liverpool, UK

Correspondence to

Dr David Hamilton;

dohamilton@doctors.org.uk

\section{ABSTRACT}

There is global concern over increasing antibiotic resistance rates due to poor antimicrobial stewardship, particularly in low-income and middle-income countries where there are limited diagnostic facilities, fewer doctors per capita and inadequate control over the production and sale of antibiotics. This quality improvement project was designed to improve the antimicrobial prescriptions practices of paramedical staff in the outpatient department of a rural district general hospital in Masanga, Sierra Leone, West Africa.

At baseline, 57 of 66 (86\%) of patients were prescribed at least one antimicrobial. On further review of 243 prescriptions for 128 patients, only $161(66 \%)$ antimicrobials were deemed appropriate for the named diagnosis when compared with international guidelines or senior medical opinion, and 86 (35\%) prescriptions had the correct drug, dose and course-length.

A full, empirical antimicrobial guideline was written and introduced to local staff via a number of different methods, including: one-to-one feedback, announcements in general meetings and printed copies placed in each outpatient room. After the first cycle, the choice of appropriate antimicrobial had improved to $85 \%$ and the correct drug, dose and course-length to $53 \%$. Unfortunately, 2 months after the second cycle, coinciding with the departure of the international internal medical physician, the rates had degraded to $65 \%$ and $43 \%$, respectively.

This study shows that implementing an empirical antimicrobial guideline can be effective at improving appropriate antibiotic prescription but that other measures are required for sustainable change. It is suggested that projects designed to change practice in low-resource countries should include national staff from the outset to improve longer term sustainability.

\section{PROBLEM}

Masanga Hospital is a non-governmental, rural district general hospital in the Tonkolili District of Sierra Leone, West Africa, serving a population of around $400000 .{ }^{1}$ It is a 100 -bed secondary-care hospital which provides paediatric, obstetric, adult internal medical and general surgical care. There is also a busy outpatient department (OPD) which treats around 500 patients per month. There is a good laboratory service for the setting; however, there is no capacity to provide microbiological culture or drug resistance testing. Services and drugs are provided for a small user-fee with most of the running costs supported by international donors.

Due to the shortage of medical staff in the country, much of the service nationwide is run by paramedical staff such as community health officers (CHOs), who have received 3 years' condensed medical education and provide frontline medical service to most rural areas. ${ }^{2}$ They are recognised by the Department of Health of Sierra Leone and can legally provide medical care, prescribe medicine and are even eligible for higher surgical training. Masanga Hospital also relies on CHOs to provide care, although clinical oversight is provided by international volunteers including doctors, nurses and pharmacists.

Sierra Leone has suffered over the past three decades from a prolonged civil war (1991-2002) and then from an epidemic of Ebola virus disease (2014-2016), and this is reflected in its healthcare system, regarded as one of the least developed in the world. For example, the latest WHO statistics show it has the highest rate of maternal mortality and also of premature deaths from non-communicable disease worldwide. ${ }^{3}$

Healthcare in the country is typically fragmented with care provided by private hospitals, governmental hospitals and international non-governmental organisations. Paediatric and maternal healthcare is provided by the government free of charge but all other care, including at governmental facilities, requires a payment from the end-user. Medication can also be bought at so-called 'pharmacies' where patrons do not require a prescription and shop-keepers will have no clinical training.

With concerns over antimicrobial resistance, irresponsible use of drugs, limited training of the paramedical staff in antimicrobial stewardship and the lack of basic bacteriological diagnostic facilities, improvements to antimicrobial prescribing were targeted. It 
was believed that the implementation of a written antimicrobial guideline would improve prescribing at the hospital. We designed a quality improvement project to improve antimicrobial prescribing using a plan do study act (PDSA) model where we would study the effect of introducing such a guideline to the hospital.

The following Specific, Measurable, Attainable, Relevant, Time-based (SMART) objectives were conceived:

To create an empirical antimicrobial guideline appropriate for the setting.

To improve antimicrobial prescription in the OPD with the correct drug with the correct course-length and dose to $70 \%$ within 6 months.

The OPD was identified as an area of particular interest because it had the least amount of medical supervision compared with the rest of the hospital, where typically inpatients were reviewed daily by physicians. It was also noted in preliminary data collection at the beginning of the project that 57 out of $66(86 \%)$ patients seen in the OPD in 1 week were prescribed at least one antimicrobial, even occasionally for non-infective diagnoses, and with the large number of patients being seen per month, even small gains proportionally would have large effects in terms of numbers.

\section{BACKGROUND}

It is well established that irresponsible antibiotic use leads to increased antibiotic resistance, increased adverse effects from medication and poorer clinical outcomes. ${ }^{4}$ Antimicrobial stewardship is a systematic method of improving antibiotic use while maintaining good individual care and is often also associated with better resource management. ${ }^{5}$ Several global actors, including the WHO, have made antimicrobial stewardship a top priority. ${ }^{6}$

Studies from the high-income countries suggest that antibiotics are used in around $10 \%-15 \%$ of outpatient consultations ${ }^{7}$ and that they are inappropriately prescribed $10 \%-30 \%$ of the time. ${ }^{78}$

Antibiotic stewardship in low-income and middle-income countries (LMICs) faces even more challenges where there are limited diagnostic facilities, fewer doctors per capita and inadequate control over the production and sale of antibiotics. ${ }^{9}$

There is a comparative paucity of data on the appropriateness of antibiotic use in LMICs. A Ghanaian study suggested it was 'not satisfactory' as evidenced by a high proportion of patients in their OPD receiving antibiotics $(55 \%)$ and the fact that the mean number of drugs prescribed was 3.5 with only $10 \%$ of their patients undergoing appropriate diagnostic testing. ${ }^{10}$ A Nigerian study quoted a similar figure of $51 \%$ of patients receiving antibiotics in the OPD. ${ }^{11}$ However it is difficult to know if simply the higher rates of antibiotic prescription compared with the developed world automatically means a higher rate of inappropriate prescription given the difference in proportion between communicable and non-communicable disease. ${ }^{12}$ The Global Health Data Exchange data from 2016 shows deaths and disability-adjusted life years from communicable disease in low-income countries at around $60 \%$ compared with 5\% in high-income countries where non-communicable disease predominates. ${ }^{13}$

It has been suggested that core elements of an antibiotic stewardship programme include: establishing standards for antibiotic prescription (including the correct selection of agent), identifying high-priority conditions where prescription is frequently inaccurate, providing individualised feedback and regularly auditing antibiotic prescription rates and outcomes. ${ }^{4514}$

\section{MEASUREMENT}

The output data were defined as the appropriateness of the choice of antimicrobial and whether the course length and drug dose were correct as per international guidelines and the British National Formulary (BNF). The data were gathered from the outpatient books where CHOs would detail their brief history, examination, laboratory results, differential diagnosis and prescribe treatment.

Anonymised data were collected prospectively for a 2 week period in February 2018 on all outpatients who were prescribed antimicrobials and was judged against the Médecins Sans Frontières (MSF) guidelines found on their smartphone app or senior clinician opinion where those guidelines were not applicable (for example, for a condition not covered by the guidelines).

One hundred and twenty-eight outpatients were prescribed a total of 251 antimicrobials (1.96 per patient), of which 243 had adequate documentation to judge the appropriateness of the prescriptions. In total, 161 (66\%) of the antimicrobial choices were deemed appropriate when compared with the above references and 86 (35\%) of those were for the correct dose and course length.

The most common prescriptions were for typhoid fever, malaria, Helicobacter pylori eradication and helminthiasis.

\section{DESIGN}

It is almost universal in hospitals in high-income counties to have a written antimicrobial guideline to aid clinicians in managing infections and promote antimicrobial stewardship. ${ }^{514}$ These are usually based on local sensitivity patterns which is not possible in many regions in LMICs where there is limited access to microbiological data, hence empirical guidelines must be used.

A 15-page antimicrobial guideline was written over the course of 2 months using a variety of sources, including: MSF guidelines; the BNF; draft antibiotic guidelines from the tertiary referral hospital in the capital, Connaught Hospital in Freetown (now available via the EMGuidance smartphone app); the website UpToDate; and discussions with Consultant Microbiologists based back in the UK. These guidelines were collated and adapted to the local setting based around drug availability and cost. They were comprehensive covering 54 different diagnoses with 31 different antimicrobials. They included adult and paediatric conditions, non-bacterial infections and guidelines 
for use in pregnancy along with a small amount of clinical advice for certain drugs or conditions. They did cover conditions that would typically require inpatient admission and were used on the wards, although this study did not assess whether the use in inpatients was effective. Later they also included advice for prophylaxis of infection for seven surgical procedures.

The guidelines were empirical by nature as there was a complete lack of microbiological data for the setting.

The advantage of a written guideline in this setting in particular is that it is more sustainable than relying on the knowledge of short-term international volunteers, and it can also be updated as drug availability changes in the area.

Judgement of the appropriateness of the drug regimen was based solely on the named diagnoses in the OPD book and no judgement on the appropriateness of the diagnosis based on the supplied clinical information was made. It was assessed against the guidelines, once written, rather than on patient outcome, a difficult thing to measure in the community in rural sub-Saharan Africa

\section{STRATEGY}

Improvement of the guidelines

After the first draft was developed as described above, it was circulated among the four other international doctors and one pharmacist working in Masanga at the time for review.

It was at this time that a number of obstetric and gynaecological diagnoses and treatments were added, and the surgical prophylaxis section was also created in conjunction with the lead surgeons. There was valuable feedback from the pharmacist in suggesting cheaper alternatives or advising when specific preparations were unavailable, such as the nitrofurantoin not being modified-release and therefore four times per day dosing was required rather than the twice per day recommended in the draft.

The guidelines were edited, reformatted and sent for a second review to general consensus. Later, during the testing phase of the guidelines, national CHOs also made other suggestions for drug dosing based on better understanding of cultural sensitivities. For example, the recommended giardiasis treatment had been $2 \mathrm{~g}$ metronidazole once daily for 3 days as per the MSF guidelines, however it was noted that patients from the area were refusing to take $10 \times 200 \mathrm{mg}$ tablets all at once, so the recommended treatment was changed to $500 \mathrm{mg}$ twice daily for 5 days.

\section{Implementing and testing the guidelines}

PDSA 1: Once the guidelines had been written, they were introduced on a Monday morning general handover where all medical, paramedical and pharmacy staff were present. There was an explanation on the importance of antimicrobial stewardship and everyone was advised to consult the guidelines regularly during prescribing.

It was also at this time when the author sat down individually with the CHOs from the OPD to review a sample of their patients' books to discuss documentation, the problems of over-prescribing and some 'low hanging fruits' that had been identified as suboptimal during the baseline measurement such as the correct H.pylori eradication dosing and typhoid fever treatment. This was important as clinicians often believe they already know certain things and will consequently not look things up that they think they are prescribing accurately. They were also given a printed copy of the guideline to take to review and keep in their OPD consultation room to examine whenever required.

After 1 week with the guidelines, another 2-week period (in March 2018) was used to prospectively collect data to assess their utility. One hundred and thirty-nine patients were prescribed a total of 283 antimicrobials, of which $241(85 \%)$ were appropriate when judged against the new local guidelines and 151 (53\%) of those were also for the correct dose and course length.

PDSA 2: After the results of PDSA 1 it was felt the guidelines were successful in improving antimicrobial prescribing so it was decided to make them more accessible and durable. Five copies were printed and placed in plastic wallet holders in large, labelled folders in each of the OPD consultation rooms, the pharmacy, the emergency ward and the physicians' office. A soft copy was also added to the computer in the seminar room and the CHOs were encouraged to download it onto their devices for portability. This was actively embraced by the CHOs who preferred to have these guidelines accessible on their phones or tablets rather than in a folder.

A copy of the surgical prophylaxis section was also printed, highlighted and placed in a plastic wallet in theatres on the wall by the table where the surgeons congregated. It is hoped that this reminded the surgical team to think carefully about antibiotic prophylaxis, although this study did not collect data to examine this.

It was also at this time when it was decided by the medical staff to increase the amount of clinical supervision of the OPD and timetables were changed to have physicians present there for an hour each day after lunch to review more complex cases. While this change was not specifically to improve antimicrobial prescription alone, it was in large part caused by this project as it was highlighted to the medical staff how little attention the OPD was receiving in comparison to the inpatient service. However, it should also be noted that doctors rarely gave specific dosing or course length instructions, especially on common infections, trusting the CHOs to either know them or look them up.

The data following PDSA 2 were collected by a UK medical student over the period of a week who was visiting Masanga 2 months (in May 2018) after the internal medical physician departed. This was done to examine whether the gains were sustainable without the daily presence of an internal medical volunteer. 


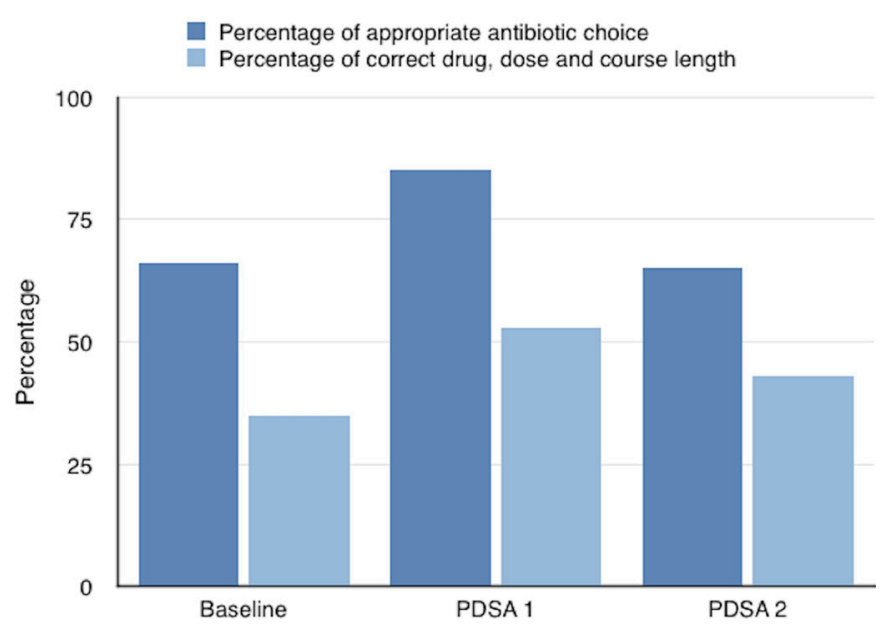

Figure 1 Appropriateness of prescribing per plan do study act (PDSA) cycle.

\section{RESULTS}

At baseline there were 243 prescriptions with 161 (66\%) of the antimicrobial choices deemed appropriate and 86 $(35 \%)$ of those were fully correct with the correct drug, dose and course-length.

After PDSA 1, this increased to 241 appropriate antimicrobials out of 283 prescriptions (85\%) with 151 of those also with the correct dose and course length (53\% of all prescriptions).

After PDSA 2, unfortunately this had fallen to near baseline with 126 of $194(65 \%)$ appropriate antimicrobial choices with 84 (43\%) fully correct.
These results are shown below in a histogram (figure 1).

The run chart in figure 2 details a timeline of the study with the results, highlighting the deteriorating appropriateness of antimicrobial prescription after the departure of the medical volunteer.

It was also noted that 85 of the $284(30 \%)$ patients in the baseline group and PDSA 1 combined were diagnosed with typhoid fever, by far the most common diagnosis. In contrast, malaria and presumed $H$. pylori infection (when a patient presented with gastro-oesophageal reflux disease) only made up $17 \%$ and $14 \%$ of diagnoses, respectively (patients commonly had more than one diagnosis given). When the CHOs were asked to guess the most common diagnosis in the OPD, interestingly typhoid fever was not in their first five responses. It would be useful to study whether these diagnoses were all appropriate as another way to limit over-prescribing.

\section{LESSONS AND LIMITATIONS}

This study was successful in introducing a local antimicrobial guideline. This is essential in a setting with limited drug availability given that drugs in international guidelines may not be available, especially when the rate of antimicrobial prescribing was higher than found elsewhere in the literature. These empirical guidelines can be useful until the healthcare facility is sufficiently advanced to offer local microbiological data. Plans for basic microbiological facilities are under way, and it is hoped that soon these guidelines will be supplemented with local

$$
\text { - Percentage of appropriate antibiotic choice }
$$

- Percentage of correct drug, dose and course length

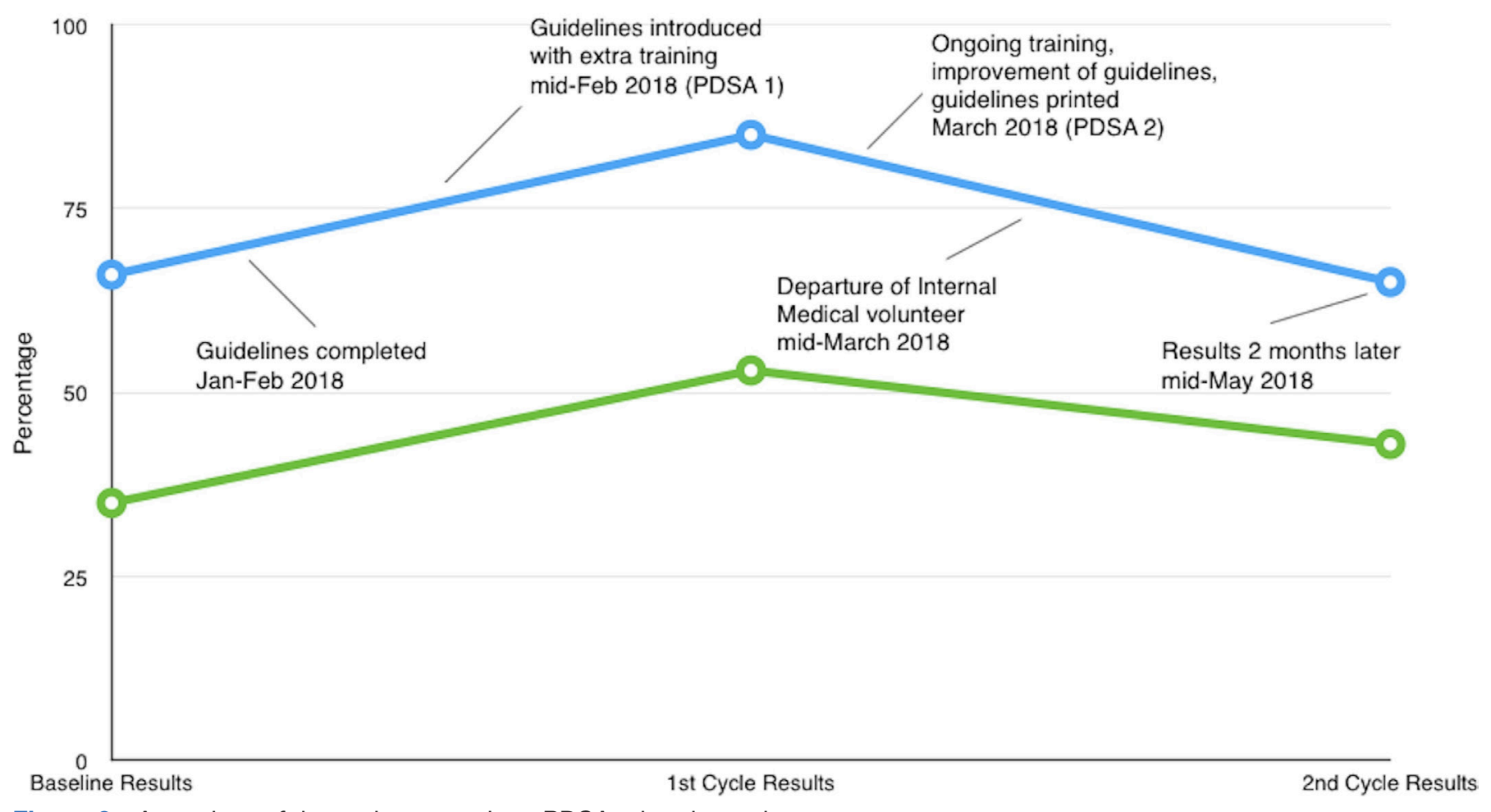

Figure 2 A runchart of the project over time. PDSA, plan do study act. 
resistance patterns, a crucial part of antimicrobial stewardship. ${ }^{4}$

The target for the project was for prescription rates of $70 \%$ having the correct drug, dose and course-length. This figure was based on developed world data suggesting up to $30 \%$ of antibiotic prescriptions may be inappropriate. ${ }^{7}$ This target was unfortunately not realised by the end of the project.

There were initial gains made by the introduction of the guidelines and some both general and individual training on good antibiotic prescribing (between baseline measurement and PDSA 1), but these gains were volatile with prescribing rates falling close to baseline level within 2 months of PDSA 2 and the departure of the internal medical physician.

The presumed cause of the deteriorating standards was the time period of the intervention was not sufficiently long enough to provide lasting change in clinician behaviour and that regrettably it would seem a written guideline was in itself not enough to reinforce change.

Literature on international health projects suggests that sustainability is important to consider from the outset, including liaising closely with local staff throughout and creating a handover process early. ${ }^{15}$ Ideally, national staff would have been involved from the initial planning stage to improve ownership of the project. It is likely this would help avoid the problem of deteriorating standards following the departure of international staff. At present, there is no specific person allocated to update these guidelines as drug availability or international recommendations change, although an editable soft copy was given to the medical superintendent of the hospital. The concept of a local 'champion' of a project features heavily in research on sustainability, ${ }^{15} 16$ and one should have been identified formally. An important role for such a person would be to continually reaudit prescribing practices against these new guidelines and give regular updates to each individual on their use of antimicrobials.

During the project, there was a focus on both individual and group training in improved antimicrobial prescribing. After discussion with the CHOs, it was clear this is not a concept that they had been trained on during their studies, and it is a significant element in improved antimicrobial stewardship. ${ }^{4}$ Yet, this educational aspect also requires regular input from someone with expertise $^{16}$ as this knowledge can degrade over time, especially if there is high staff turnover.

While the study was not investigating the accuracy of diagnosis of the OPD, this may be relevant as perhaps the drug regimen prescribed may have been effective, even if not correct for the diagnosis given. Further study by using patient outcome as a marker for success of prescription or examining the accuracy of diagnoses would be very useful, although challenging in this setting.

The first author was an international volunteer only in post for 6 months, and it took a number of months for the problem to be initially identified as a particular issue. If a similar project were to be done in another area in
sub-Saharan Africa, there were some useful ready-made antibiotic guidelines on the EMGuidance smartphone app that had not been published at the time of this project was started.

A potential source for bias is that the same clinician wrote the guidelines and reviewed the prescriptions for appropriateness. An attempt to limit this bias was done by ensuring that all decisions on appropriateness were taken directly from the written guidelines and consistent throughout the cycles.

\section{CONCLUSION}

Antibiotic stewardship is particularly difficult to perform in the LMICs with limited access to healthcare facilities, trained staff and microbiological data, therefore strategies to improve this are of vital importance in safeguarding future drugs. This study has shown that implementing an empirical antimicrobial guideline can be effective at improving appropriate antibiotic prescription in a low-resource setting, although lasting change can be difficult to implement and factors leading to long-term sustainability of such an intervention remain to be identified. It is vital to remember that any projects designed to change practice in such countries include national staff to improve sustainability and embracement of the project locally.

Acknowledgements We would like to thank Dr Jon Vas Nunes, Dr Hanna Matheron, Dr Daniel van Leerdam, Dr Martelien Grootjans and Simon Christensen for their input on the writing of the guidelines. We would also like to thank Professor $\mathrm{M}$ Grobusch for his thoughts on the project. Finally, we would like to thank the staff of Masanga Hospital for their warmth and assistance during our time there.

Contributors DH designed the study, collated data and drafted the original manuscript. IB collated data and helped revise the manuscript. All authors gave final approval of the of the version published. DH is responsible for the overall content as guarantor.

Funding The authors have not declared a specific grant for this research from any funding agency in the public, commercial or not-for-profit sectors.

Competing interests None declared.

Patient consent for publication Not required.

Provenance and peer review Not commissioned; externally peer reviewed.

Open access This is an open access article distributed in accordance with the Creative Commons Attribution Non Commercial (CC BY-NC 4.0) license, which permits others to distribute, remix, adapt, build upon this work non-commercially, and license their derivative works on different terms, provided the original work is properly cited, appropriate credit is given, any changes made indicated, and the use is non-commercial. See: http://creativecommons.org/licenses/by-nc/4.0/.

\section{REFERENCES}

1. García-Tardón N, Gresnigt TM, Fofanah AB, et al. Hepatitis B and C in tonkolili province, sierra leone. Lancet 2017;390:1485.

2. van Duinen A, Cobb N, Mansaray A, et al. Sierra Leone's Community Health Officers: a resilient, responsive health workforce. 2017 https:// whoeducationguidelines.org/blog/sierra-leone\%E2\%80\%99scommunity-health-officers-resilient-responsive-health-workforce.

3. Global Health Observatory Data. 2015 http://www.who.int/gho/ncd/ en/.

4. NICE guidelines [NG15]. Antimicrobial stewardship: systems and processes for effective antimicrobial medicine use. $2015 \mathrm{https}: / /$ www.nice.org.uk/guidance/ng15/chapter/1-Recommendations.

5. Holubar M, Deresinski S. Baron EAntimicrobial stewardship in outpatient settings. , UpToDate, 2017. https://www.uptodate. 
com/contents/antimicrobial-stewardship-in-outpatient-settings Subscription Required.

6. Leung E, Weil DE, Raviglione M, et al. The WHO policy package to combat antimicrobial resistance. Bull World Health Organ 2011;89:390-2.

7. Fleming-Dutra KE, Hersh AL, Shapiro DJ, et al. Prevalence of Inappropriate Antibiotic Prescriptions Among US Ambulatory Care Visits, 2010-2011. JAMA 2016;315:1864-73.

8. Smieszek T, Pouwels KB, Dolk FCK, et al. Potential for reducing inappropriate antibiotic prescribing in English primary care. $J$ Antimicrob Chemother 2018;73:ii36-ii43.

9. Ayukekbong JA, Ntemgwa M, Atabe AN. The threat of antimicrobial resistance in developing countries: causes and control strategies. Antimicrob Resist Infect Control 2017;6:47.

10. Prah J, Kizzie-Hayford J, Walker E, et al. Antibiotic prescription pattern in a Ghanaian primary health care facility. Pan Afr Med $J$ 2017;28:214
11. Ibrahim M. Physicians' prescribing behaviour in two tertiary health care facilities in north-western Nigeria - Analysis of 518 prescriptions. Sahel Med J 2004;7:115-8.

12. World Health Organisation. Burden of disease: DALYs. $2004 \mathrm{https} / / /$ www.who.int/healthinfo/global_burden_disease/GBD_report_ 2004update_part4.pdf.

13. Global Health Data Exchange. Global Burden of Disease Results Tool. $2018 \mathrm{http}: / / g h d x$.healthdata.org/gbd-results-tool.

14. Ashiru-Oredope D, Sharland M, Charani E, et al. Improving the quality of antibiotic prescribing in the nhs by developing a new antimicrobial stewardship programme: start smart--then focus. $J$ Antimicrob Chemother 2012;67:i51-i63.

15. Edwards NC, Roelofs SM. Sustainability: the elusive dimension of international health projects. Can J Public Health 2006;97:45-9.

16. Johnson $\mathrm{K}$, Hays $\mathrm{C}$, Center $\mathrm{H}$, et al. Building capacity and sustainable prevention innovations: a sustainability planning model. Eval Program Plann 2004;27:135-49. 